\title{
FIFTH ANNUAL MAY DAY CENSUS, REGINA
}

On May 15, 1965 teams from the Regina Natural History Society spent the day in the field making a census of birds seen in the Regina district, an area bounded by a circle 30 miles in diameter (defined for the Birds of Regina, 1961). On May 14, the day preceding the count, great numbers of warblers and particularly of shorebirds were noted by R. W. Nero and R. R. Taylor, but the May 15 count produced much reduced numbers of these species, indicating that there had been a large migration out of the area on the evening of May 14-15. Nevertheless, 143 species were observed.

SPECIES LIST : Red-necked Grebe (2), Horned Grebe (19), Eared Grebe (135), Western Grebe (24), Pied-billed Grebe (3), Double-crested Cormorant (1), Great Blue Heron (5), Blackcrowned Night Heron (3), Mute Swan (5), Whistling Swan (11), Trumpeter Swan (1), Canada Goose (331), Mallard (293), Gadwall (71), Pintail (185), Green-winged Teal (13), Blue-winged Teal (157), Cinnamon Teal (2), American Widgeon (83), Shoveler (85), Redhead (53), Ring-necked Duck (2), Canvasback (78), Lesser Scaup (213), Common Goldeneye (2), Bufflehead (3), Ruddy Duck (61), Redbreasted Merganser (1), Sharp-shinned Hawk (1), Red-tailed Hawk (11), Swainson's Hawk (5), Marsh Hawk (23), Pigeon Hawk (1), Sparrow Hawk (1), Ring-necked Pheasant (3), Gray Partridge (11), Virginia Rail (1), Sora (16), American Coot (311), Semipalmated Plover (3), Killdeer (153), American Golden Plover (62), Spotted Sandpiper (19), Solitary Sandpiper (1), Willet (49), Greater Yellowlegs (1), Lesser Yellowlegs (12), Pectoral Sandpiper (137), Baird's Sandpiper (266), Least Sandpiper (36), Dowitcher (species?) (38), Semipalmated Sandpiper (10), Marbled Godwit (25), Hudsonian Godwit (2), Sanderling (12), American Avocet (30), Wilson's Phalarope (43), California Gull (2), Ring-billed Gull (78), Franklin's Gull (24), Common Tern (81), Black Tern (375), Rock Dove (64), Mourning Dove (126), Great Horned Owl (10), Belted
Kingfisher (7), Yellow-shafted Flicker (21), Red-shafted Flicker (1), Yellow-bellied Sapsucker (1), Hairy Woodpecker (2), Downy Woodpecker (2), Eastern Kingbird (11), Western Kingbird (44), Say's Phoebe (2), Traill's Flycatcher (1), Least Flycatcher (probably includes other empidonax flycatchers) (39), Western Wood Pewee (3), Horned Lark (900), Tree Swallow (48), Bank Swallow (29), Barn Swallow (50), Purple Martin (35), Blackbilled Magpie (55), Common Crow (193), House Wren (16), Rock Wren (1), Catbird (2), Brown Thrasher (70), Robin (164), Hermit Thrush (3), Swainson's Thrush (53), Graycheeked Thrush (15), Veery (15), Mountain Bluebird (1), Ruby-crowned Kinglet (1), Sprague's Pipit (5), Loggerhead Shrike (16), Starling (28), Philadelphia Vireo (2), Warbling Vireo (25), Black-and-white Warbler (4), Tennessee Warbler (2), Orange-crowned Warbler (13), Yellow Warbler (40), Magnolia Warbler (1), Cape May Warbler (1), Myrtle Warbler (132), Bay-breasted Warbler (1), Blackpoll Warbler (18), Palm Warbler (5), Ovenbird (8), Northern Waterthrush (50), Yellowthroat (5), House Sparrow $(1000+)$, Western Meadowlark (307), Yellow-headed Blackbird (227), Red-winged Blackbird (1077), Baltimore Oriole (29), Brewer's Blackbird (442), Common Grackle (118), Brown-headed Cowbird (146), Rose-breasted Grosbeak (13), Purple Finch (1), American Goldfinch (12), Rufous-sided Towhee (9), Lark Bunting (13), Savannah Sparrow (79), Baird's Sparrow (1), Sharp-tailed Sparrow (2), Vesper Sparrow (53), Lark Sparrow (2), Slate-colored Junco (2), Chipping Sparrow (542), Clay-colored Sparrow (485), Harris' Sparrow (33), Whitecrowned Sparrow (92), White-throated Sparrow (142), Lincoln's Sparrow (47), Swamp Sparrow (4), Song Sparrow (35), McCown's Longspur (46), Lapland Longspur (10,000士), Chestnut-collared Longspur (310).

Of special interest for the area was the sighting of the two Cinnamon Teal ( $\sigma^{7}$ and $\%$ ) on the Boggy Creek below the Condie Dam, by R. W. Nero and R. R. Taylor-Frank H. Brazier, compiler.

\section{NINTH ANNUAL MAY DAY CENSUS, SASKATOON}

On May 15, 1965 the Ninth Annual Saskatoon May Day census was held. Groups of members of the Saskatoon Natural History Society, under the co-ordination of Jim Slimmon, participated in the dawn to dusk count. A total of 22 people spent 247 man-hours in the field and counted 137 species.

Two new species were added to the Saskatoon list during the count. The Short-billed Marsh Wren was a first, found by J. Hogg. The Smith's Long- 
spur was also a first; five were seen by Stuart and Mary Houston and Jonathan Gerrard.

SPECIES LIST: Red-necked Grebe, Horned Grebe, Eared Grebe, Western Grebe, Pied-billed Grebe, White Pelican, Whistling Swan, Canada Goose, White-fronted Goose, Mallard, Gadwall, Pintail, Green-winged Teal, Blue-winged Teal, American Widgeon, Shoveler, Redhead, Ringnecked Duck, Canvasback, Lesser Scaup, Common Goldeneye, Bufflehead, Ruddy Duck, Cooper's Hawk, Red-tailed Hawk, Swainson's Hawk, Ferruginous Hawk, Marsh Hawk, Peregrine Falcon, Sparrow Hawk, Ruffed Grouse, Sharp-tailed Grouse, Gray Partridge, Sandhill Crane, Sora, American Coot, Semipalmated Plover, Killdeer, American Golden Plover, Black-bellied Plover, Ruddy Turnstone, Common Snipe, Long-billed Curlew, Upland Plover, Spotted Sandpiper, Solitary Sandpiper, Willet, Lesser Yellowlegs, Pectoral Sandpiper, Baird's Sandpiper, Least Sandpiper, Stilt Sandpiper, Semipalmated Sandpiper, Marbled Godwit, Sanderling, American Avocet, Wilson's Phalarope, Northern Phalarope, California Gull, Ring-billed Gull, Franklin's Gull, Common Tern, Black Tern, Rock Dove, Mourning Dove, Burrowing Owl, Common Nighthawk, Yellow- shafted Flicker, Yellow - bellied Sapsucker, Hairy Woodpecker, Downy Woodpecker, Eastern Kingbird, Western Kingbird, Eastern Phoebe, Least Flycatcher, Horned Lark, Tree Swallow, Bank Swallow, Barn Swallow, Purple Martin, Blue Jay, Black-billed Magpie, Common Crow, Black-capped Chickadee, Brown Creeper, House Wren, Short-billed Marsh Wren, Catbird, Brown Thrasher, Robin, Swainson's Thrush, Gray-cheeked Thrush, Veery, Mountain Bluebird, Ruby-crowned Kinglet, Water Pipit, Sprague's Pipit, Loggerhead Shrike, Starling, Black-and-white Warbler, Tennessee Warbler, Orange-crowned Warbler, Yellow Warbler, Myrtle Warbler, Blackpoll Warbler, Bay-breasted Warbler, Palm Warbler, Ovenbird, Northern Waterthrush, Yellowthroat, Wilson's Warbler, American Redstart, House Sparrow, Western Meadowlark, Yellow-headed Blackbird, Redwinged Blackbird, Baltimore Oriole, Brewer's Blackbird, Common Grackle, Brown-headed Cowbird, Rose-breasted Grosbeak, Rufous-sided Towhee, Savannah Sparrow, Baird's Sparrow, Vesper Sparrow, Lark Sparrow, Slate-colored Junco, Chipping Sparrow, Clay-colored Sparrow, Harris' Sparrow, White-crowned Sparrow, White-throated Sparrow, Lincoln's Sparrow, Song Sparrow, Lapland Longspur, Smith's Longspur, Chestnutcollared Longspur.-Jim Slimmon, Compiler.

\section{SOME INTERESTING BIRD RECORDS FOR CENTRAL SASKATCHEWAN}

From about July 22 to August 12, 1961 Dr. Mary M. Tremaine, microbiologist on the faculty of the University of Nebraska and a well-known amateur ornithologist, travelled in Saskatchewan on a bird-finding tour. Although she made detailed notes these have since been lost. However, a few of her observations, which were reported in correspondence with the editor shortly after her trip and recently, are noteworthy even though precise data are lacking.

Wood Duck, one, Duck Lake, near Carlton.

Arctic Tern (?), five, seen flying over Montreal Lake (south of La Ronge) during a mile walk along the east lake shore. "In spite of my experience, or perhaps because of it, I will always hesitate on Arctic Terns in late summer. These had the greyer mantle, greyer side of head, red bills, but with some black. I think they were Arctics, but who knows?" (pers. corres. to R. Nero, August 16, 1961). "I questioned the Arctic Terns because they didn't perch and I couldn't verify the short legs ... We had them on Cape Cod and Nova Scotia, too, so I've had pretty good looks at them." (pers. corres. to R. Nero, 1965).

Caspian Tern, one, flying over Montreal Lake.

Barred Owl, one, Little Sandy Lake, near MacDowall.

Great Gray Owl (?), two, near Torch River. "I saw two very large owls at night on a bridge rail - not Great Horned and not Barred - absolutely immense. I thought they were probably Great Gray Owls. They flew off down the road ahead of my lights and disappeared." (pers. corres. to R. Nero, 1965).

Townsend's Solitaire, one, east side of Fishing Lake, Nipawin Provincial Forest.

Evening Grosbeak, some along the road north of La Ronge.

\section{NEST RECORDS}

You are reminded that nest records for the 1965 season should be sent to the Prairie Nest Records Scheme, c/o Robert R. Taylor, Box 1121, Regina. If you need more nest record cards, write to the same address. 